\title{
Users' Assessement of the Visualization with Gauges of a Guideline-Based Decision Support System Output: A Qualitative Study of Focus Groups
}

\author{
Abir ABDELLATIF,b,c, ${ }^{\mathrm{a}, \text { Jacques BOUAUD }}{ }^{\mathrm{d}, \mathrm{a}}$, Mélissa THOLOMIER ${ }^{\mathrm{e}, \mathrm{b}}$, Joël \\ BELMIN $^{\mathrm{a}, \mathrm{b}}$ and Brigitte SEROUSSI ${ }^{\mathrm{a}, \mathrm{f}}$ \\ a Sorbonne Université, Université Sorbonne Paris Nord, Inserm, UMR S_1142, \\ LIMICS, Paris, France \\ b AP-HP, Hôpital Charles-Foix, Ivry-sur-Seine, France \\ ${ }^{\mathrm{c}}$ Teranga Software, Paris, France \\ d AP-HP, DRCI, Paris, France \\ eSorbonne Université, Paris, France \\ ${ }_{\mathrm{f}}$ AP-HP, Hôpital Tenon, Paris, France
}

\begin{abstract}
Computerized decision support systems (CDSSs) are still poorly routinely implemented in clinical practices mainly because of usability problems related to the technology interface. We previously proposed to use gauges to visualize the output of a guideline-based CDSS applied to malnutrition and pressure ulcer management in nursing homes. This interface was assessed by four focus groups including 16 healthcare professionals with expertise in geriatrics. A USElike questionnaire was distributed. Participants considered the dashboard-withgauges visualization was useful (94\%), easy to use (63\%), easy to learn $(88 \%)$, and $88 \%$ thought they could be satisfied with it. However, concerns were expressed about the difficulty to follow up multiple healthcare problems.
\end{abstract}

Keywords. Clinical decision support system, Graphical users' interface, Visualization, Focus groups, Evaluation

\section{Introduction}

Although computerized decision support systems (CDSSs) have proven to improve patient care quality, they are poorly routinely implemented in clinical settings, partly because of usability problems related to the user interface [1]. In a prior work [2], we have proposed to display gauges gathered in a dashboard to represent the guideline-based recommendations issued by the NETSmart CDSS for the management of malnutrition and pressure ulcers of nursing home $(\mathrm{NH})$ residents. Focus groups (FGs) were organized to assess geriatrics healthcare professional acceptance of this proposal ${ }^{2}$.

\footnotetext{
${ }^{1}$ Corresponding Author: Abir Abdellatif, Hôpital Charles-Foix, 7 avenue de la République, 94200 Ivrysur-Seine, France. E-mail: abir.abdellatif@gmail.com

${ }^{2}$ This research is funded by the ANRT CIFRE Grant $n^{\circ} 2018 / 0307$ for AA.
} 


\section{Methods}

FG participants were recruited among the staff of Charles Foix geriatric hospital (Assistance Publique - Hôpitaux de Paris, France). The evaluation protocol was made of three steps: (i) a short tutorial to present the study context, the description of guidelinebased decision support modalities, and the introduction of the dashboard-with-gauge visualization on a simulated patient case, (ii) semi-structured discussion to question four dimensions: "Assessment of the dashboard", "Assessment of gauges", "Assessment of recommendations' display", and "Assessment of the global user interface", (iii) qualitative user assessment of usefulness, ease of use, ease of learning, and satisfaction through a short USE-derived questionnaire [3]. Discussions between participants were audio-recorded. Data were analyzed using the six steps of the thematic analysis [4]: familiarization with the data, generating initial codes, searching for themes, reviewing themes, defining and naming themes, and producing the report.

\section{Results}

There were 16 participants including six geriatricians, six nurses, two care assistants, one dietician, and one psychometrician. Most participants considered the dashboard-withgauges visualization was useful (94\%), easy to use (63\%), easy to learn (88\%), and they were globally $88 \%$ to think they could be satisfied with it. The qualitative analysis produced six themes: use of a dashboard to display patient condition, use of gauges for severity levels, modalities used to display recommendations, overall graphical interface, respect of user autonomy despite the use of a CDSS, user interaction with the CDSS (issues of interoperability with EHR data).

\section{Discussion and conclusion}

The dashboard-with-gauge visualization was well accepted but with some hesitations. Participants considered this kind of interface could improve their ability to take care of their patients. However, they had mixed feelings about the gauge to summarize patient condition considering this visualization was appropriate to follow patient evolution but didn't show the patient history. The responses to the USE-like questionnaire evidenced that the general graphical design was well accepted despite some training guidance seems to be necessary (only $63 \%$ found the visualization easy or very easy to use).

\section{References}

[1] Wipfli R, Ehrler F, Bediang G, Bétrancourt M, Lovis C. How Regrouping Alerts in Computerized Physician Order Entry Layout Influences Physicians' Prescription Behavior: Results of a Crossover Randomized Trial. JMIR Hum Factors 2016;3(1):e15.

[2] A. Abdellatif, J. Bouaud, J. Belmin, B. Seroussi, Visualization of Guideline-Based Decision Support for the Management of Pressure Ulcers in Nursing Homes, Stud Health Technol Inform. 2020;275:1-5.

[3] USE Questionnaire: Usefulness, Satisfaction, and Ease of use, Available at: https://garyperlman.com/quest/quest.cgi?form=USE, Accessed March 2021.

[4] V. Braun and V. Clarke. Using thematic analysis in psychology, Qualitative Research in Psychology 2006,3(2):77-101. 